\title{
The discovery reach for mini black holes with the ATLAS Detector at the LHC
}

\section{Michiru KANEDA*}

On behalf of ATLAS Collaboration

International Center for Elementary Particle Physics (ICEPP), University of Tokyo

E-mail: kaneda@icepp.s.u-tokyo.ac.jp

The hierarchy problem is a major weakness of the Standard Model. Theories with extra spatial dimensions have a potential to solve this problem since they bring the Planck scale close to the $\mathrm{TeV}$ scale. At the LHC, one of the interesting signals resulting from such a low Planck scale is the production of mini black holes. Here, we discuss the analysis method for detecting black holes with the ATLAS detector and the potential for discovery at the LHC. 


\section{Introduction}

The large difference between the Planck scale and the electroweak symmetry breaking scale is known as the hierarchy problem. Several approaches have been investigated for solving the problem, among which scenarios with extra dimensions, pioneered by Arkani-Hamed, Dimopoulos and Davali (ADD) [1, 2, 3], and Randall and Sundrum (RS) [4, 5]. In these models, only the gravitational field is allowed to propagate in to the bulk, which includes additional large compactified flat dimensions (ADD) or a single warped extra dimension, whereas other standard model particles are confined to our 3-brane. As a result, gravity appears very weak in our 4 dimensional space-time, but, the D-dimensional fundamental Plank scale $M_{D}$ can be as low as the TeV-scale instead of the Planck scale $M_{P l} \sim 10^{19} \mathrm{GeV}$.

If $M_{D}$ is as low as $\sim \mathrm{TeV}$, the $\mathrm{LHC}$ could generate $\mathrm{TeV}$-scale mini black holes. Observation of such black holes would yield information on extra dimensions, testing general relativity and models of quantum gravity.

There exist many direct and indirect approaches to investigate the presence of extra dimensions, using table-top and particle accelerator experiments, or from cosmic ray data and astrophysical measurements. These measurements set limits on the fundamental Planck scale of about $M_{D}>1 \mathrm{TeV}[\overline{6}]$.

In this study, the parton level cross section for black hole production assumes a semi-classical model:

$$
\hat{\sigma}_{a b \rightarrow \mathrm{BH}}=\pi r_{\mathrm{h}}^{2}
$$

where $\mathrm{a}$ and $\mathrm{b}$ are the interacting partons and $r_{h}$ is the horizon radius of the generated black hole. This assumption is valid only when the mass of the black hole $M_{B H} \gg M_{D}$. For this reason, we will limit our analysis to black holes with $M_{B H}>5 \mathrm{TeV}$ and $>8 \mathrm{TeV}$ for $M_{D}=1 \mathrm{TeV}$. Such mini black holes decay immediately $\left(\sim 10^{-26}\right.$ s), with most of its energy emitted by Hawking radiation. The event generator Charybdis [7] was used for this study. It implements the effects of grey-body factors but does not account for graviton emission, expected to be small. Details of this study can be found in $[8]$

\section{Event topology and event selection}

A black hole will emit particles of the Standard Model with a relative probability which depends only on their number of degrees of freedom, measured in quantum variables (spin, charge, color, etc (Fig. 1). A break from perfect democratic decay would result from conservation of quantum variables, from the effect of grey-body factors and from the decay in the Planck Phase.

As can be seen in Fig. 2, many particles emitted in black hole decay have high $p_{T}$. This allows reliable trigger of such events, most of which (>99\%) can be triggered by the requirement of a high $p_{T}$ single jet. The energy spectrum of emitted particles by Hawking radiation depends on the Hawking temperature, which depends on the number of extra dimensions $(n), M_{D}$ and $M_{B H}$. For higher $n$ values, the Hawking temperature is higher and therefore emitted particles have higher $p_{T}$ and the particle multiplicity is lower (Fig. 3).

A simple set of variables can be useful for distinguishing black hole events from backgrounds. In black hole events, the fourth leading particle have higher $p_{T}$ than in backgrounds. In Fig. 4 , 

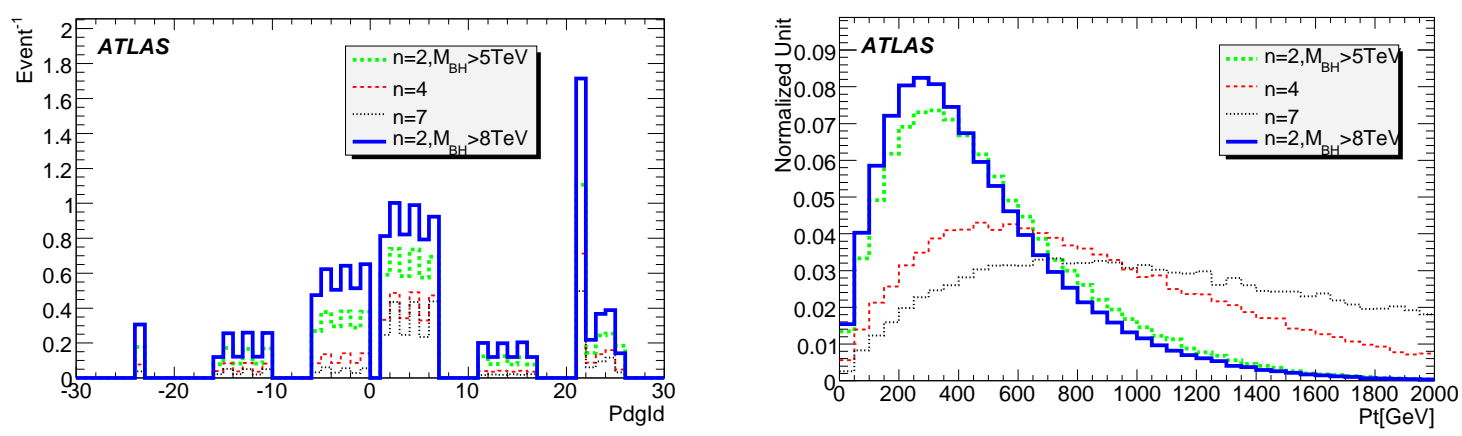

Figure 1: PDG code of particles emitted from black Figure 2: $p_{T}$ distributions of all particles emitted hole decay from the black hole

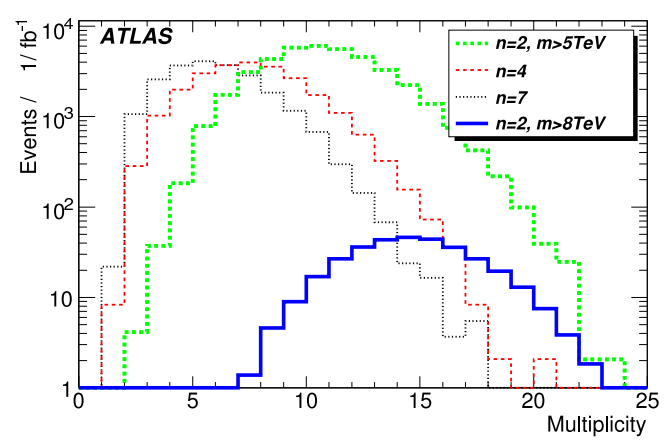

Figure 3: Particle multiplicity of black hole event

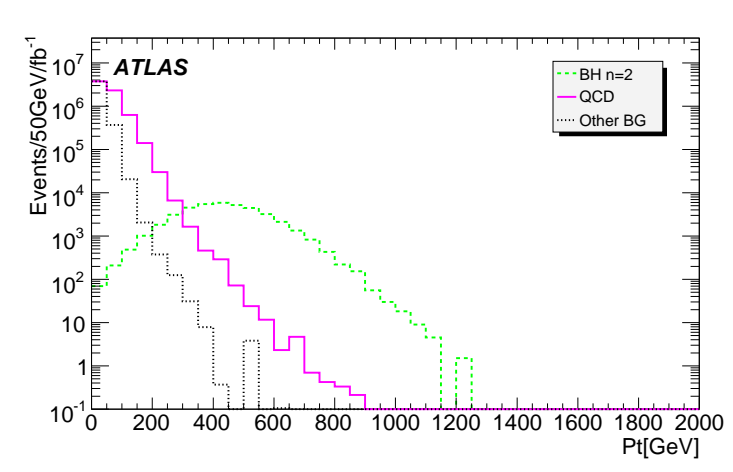

Figure 4: $p_{T}$ distributions of 4 th leading particle

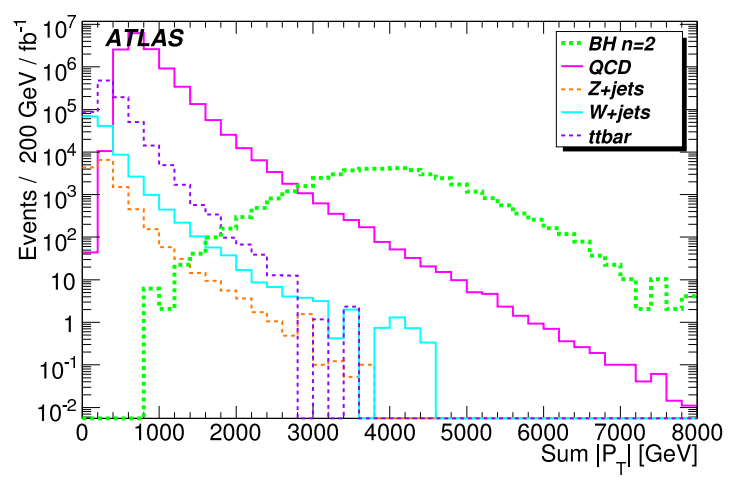

Figure 5: $\Sigma\left|p_{T}\right|$ distributions

this distribution is shown with a green thick dashed line for the black hole signal, a pink solid line for QCD multi-jet events $\_n d$ a black dotted line for other background, including $t \bar{t}$ and vector boson + jets events. Fig. 5 shows the scaler sum of $p_{T}$ of all particles in an event $\left(\Sigma\left|p_{T}\right|\right)$. With the use of these variables, two event selections were studied. One requires $\Sigma\left|p_{T}\right|>2.5 \mathrm{TeV}$ and at least one of $50 \mathrm{GeV}$ lepton (electron or muon). The requirement of high $p_{T}$ lepton especially suppresses QCD multi-jet processes. Another event selection is based on the multiplicity of high 
Table 1: Acceptance of the multi-object requirements for each dataset in $\mathrm{fb} .90 \%$ confidence limits are used when no events passed the requirements.

\begin{tabular}{ccccc}
\hline \hline Dataset & $\begin{array}{c}\text { Before selection } \\
(\mathrm{fb})\end{array}$ & $\begin{array}{c}\text { After multi-object } \\
\text { requirement }(\mathrm{fb})\end{array}$ & $\begin{array}{c}\text { After lepton requirement } \\
(\mathrm{fb})\end{array}$ & Acceptance \\
\hline$n=2, m>5 \mathrm{TeV}$ & $40.7 \times 10^{3}$ & $38.9 \pm 0.4 \times 10^{3}$ & $14.0 \pm 0.2 \times 10^{3}$ & 0.34 \\
$n=4, m>5 \mathrm{TeV}$ & $24.3 \times 10^{3}$ & $17.9 \pm 0.3 \times 10^{3}$ & $4521 \pm 126$ & 0.19 \\
$n=7, m>5 \mathrm{TeV}$ & $22.3 \times 10^{3}$ & $9953 \pm 185$ & $1956 \pm 82$ & 0.087 \\
$n=2, m>8 \mathrm{TeV}$ & 338 & $338 \pm 4$ & $164 \pm 3$ & 0.49 \\
$t \bar{t}$ & $833 \times 10^{3}$ & $129 \pm 27$ & $36_{-9}^{+12}$ & $4.3 \times 10^{-5}$ \\
QCD dijets & $12.8 \times 10^{6}$ & $38.9 \pm 1.9 \times 10^{3}$ & $6_{-3}^{+107}$ & $5.6 \times 10^{-7}$ \\
W+jets & $560 \times 10^{3}$ & $99_{-22}^{+28}$ & $56_{-13}^{+24}$ & $1 \times 10^{-3}$ \\
Z+jets & $51.8 \times 10^{3}$ & $29_{-4}^{+90}$ & $19_{-3}^{+90}$ & $4 \times 10^{-4}$ \\
\hline \hline
\end{tabular}

$p_{T}$ particles. It requires multi-object: at least four objects with $p_{T}>200 \mathrm{GeV}$, at least one of which is a lepton. The two event selections result in approximately the same efficiency and suppression of background. Here, we show the result of the multi-object selection. The result of the $\Sigma\left|p_{T}\right|$ selection and further details can be found in [8].

\section{Discovery potential}

Table 1 shows the efficiencies and rejection powers of the multi-object selection. All SM backgrounds are strongly suppressed.

Fig. 6 shows the reconstructed black hole mass distribution, obtained from the vector sum of all particle 4-momenta in events. Missing $E_{T}$ was also included as $p_{z}$ less and mass less particles to account for non-interacting particles. Since the signal has a high cross section and high acceptance, the mass distribution would reveal a clear signal.

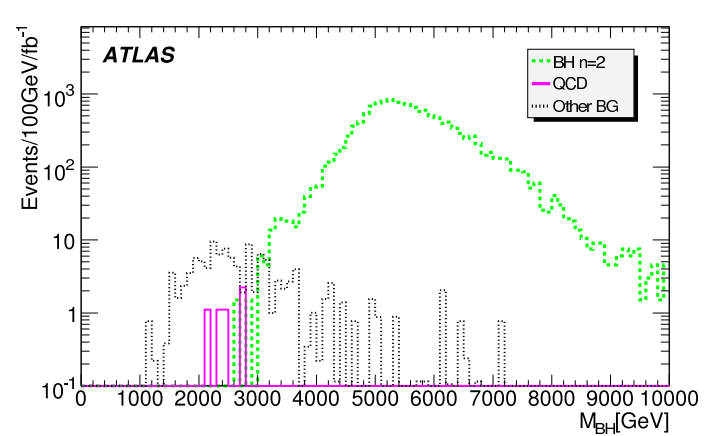

Figure 6: Reconstructed mass of black hole

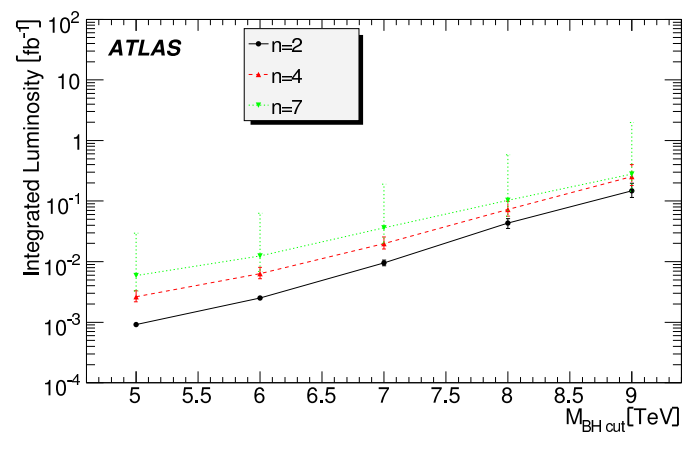

Figure 7: Discovery potential for black hole with the multi-object selection

The discovery potential was calculated as the luminosity required for a significance $S / \sqrt{B}>5$ and a minimum signal: $S>10$ (Fig. 7). Since the semi-classical model cannot describe the mass range $M_{B H} \sim M_{D}$, a cut on the minium mass of the black hole must be applied. The abcissa of Fig. 7 
refers to the value of this cut. The errors shown in the figure include statistical uncertainties and systematic uncertainties from detector performance such as the lepton identification efficiency and theoretical uncertainties from black hole production. These were evaluated by varying parameters of the Charybdis generator.

\section{Conclusions}

The observation of $\mathrm{TeV}$-scale mini black holes would provide clear evidence for the existence of extra dimensions, as predicted by some models which attempt to solve the hierarchy problem. Since the LHC is a TeV scale accelerator, it has the potential of producing them. We have studied methods to detect such mini black holes with the ATLAS detector. If the semi classical cross section estimations are valid, only a few $\mathrm{pb}^{-1}$ are needed to discover black hole of minimum mass of $5 \mathrm{TeV}$ [for $M_{D}=1 \mathrm{TeV}$ ]. With $1 \mathrm{fb}^{-1}, 8 \mathrm{TeV}$ black holes could be discovered. Methods to measure the information of extra dimensions such $n$ or $M_{D}$ were also investigated. Details of these could be found in [8].

\section{References}

[1] N. Arkani-Hamed, S. Dimopoulos and G. Dvali, Phys. Lett. B 429 (1998) 263; arXiv:hep-ph/9803315.

[2] I. Antoniadis, N. Arkani-Hamed, S. Dimopoulos and G. Dvali, Phys. Lett. B 436 (1998) 257; arXiv:hep-ph/9804398.

[3] N. Arkani-Hamed, S. Dimopoulos and G. Dvali, Phys. Rev. D 59 (1999) 086004; arXiv:hep-ph/9807344.

[4] L. Randall and R. Sundrum, Phys. Rev. Lett. 83 (1999) 3370; arXiv:hep-ph/9905221.

[5] L. Randall and R. Sundrum, Phys. Rev. Lett. 83 (1999) 4690; arXiv:hep-th/9906064.

[6] C. Amsler, et al. Phys. Lett. B 667 (2008) 1

[7] C. M. Harris, P. Richardson and B. R. Webber, J. High Energy Phys. 0308 (2003) 033; arXiv:hep-ph/0307305.

[8] The ATLAS Collaboration, CERN-OPEN-2008-020, Geneva, 2008, to appear. 